\title{
Desarrollo de una herramienta de software sistema administración de órdenes e inventario (SAO-DiTOTINOS) para restauran DiTOTINOS
}

\section{Development of a software tool system administration of orders and inventory (SAO-DiTOTINOS) to restore DiTOTINOS}

\author{
VEGA-CHÁVEZ, Efrén †*, GUTIÉRREZ-TORRES, Luis Germán y MARTÍNEZ-LÓPEZ, Fernando \\ José
}

Instituto Tecnológico Superior del Sur de Guanajuato. Educación Superior 2000, Benito Juárez, 38980 Uriangato, Gto.

ID $1^{\mathrm{er}}$ Autor: Efrén, Vega-Chávez / ORC ID: 0000-0001-8918-3170

ID $2^{\text {do }}$ Coautor: Luis Germán, Gutiérrez-Torres / ORC ID: 0000-0001-7912-2147

ID $3^{\text {er }}$ Coautor: Fernando José, Martínez-López / ORC ID: 0000-0001-9781-1800

DOI: $10.35429 / J A D .2019 .8 .3 .23 .28$

Recibido 05 de Abril, 2019; Aceptado 30 de Junio, 2019

\begin{abstract}
Resumen
El presente describe los resultados de las dos primeras fases de la investigación que se plantearon con la finalidad de dar una solución al restaurante DiTotinos que se encuentra en el municipio de Moroleón del estado de Guanajuato. La problemática principal a tratar es la forma en la cual se realizaba la actividad de reporteo de productos disponibles en el almacén, así como dar seguimiento a las órdenes presenciales y/o por teléfono, las cuales se registraban de manera manual, es por ello que se sugirió el desarrollo de la herramienta de software Sistema Administración de Órdenes e Inventario DiTOTINOS (SAO-DiTOTINOS). Dicho software tendrá la función de controlar las entradas y salidas de los productos del inventario y administrar las órdenes, para lo cual se aplicó la siguiente metodología: fase I, Desarrollo de las herramientas de software (usando metodología de desarrollo lineal secuencial), fase II, Implementar la herramienta de software. Con lo anterior el beneficiado directo es la empresa restaurant DiTotinos principalmente el área de órdenes e inventario.
\end{abstract}

Software, Inventario, Órdenes

\begin{abstract}
The present describes the results of the first two phases of the investigation that were proposed in order to provide a solution to the DiTotinos restaurant located in the municipality of Moroleón in the state of Guanajuato. The main problem to be treated is the way in which the activity of reporting products available in the warehouse was carried out, as well as following up on the face-toface and / or telephone orders, which were registered manually, which is why the development of the software tool System Administration of Orders and Inventory DiTOTINOS (SAO-DiTOTINOS) was suggested. This software will have the function of controlling the entries and exits of the products of the inventory and administer the orders, for which the following methodology was applied: phase I, Development of software tools (using sequential linear development methodology), phase II, Implement the software tool. With this, the direct beneficiary is the restaurant company DiTotinos, mainly the order and inventory area.
\end{abstract}

Software, Inventory, Orders

Citación: VEGA-CHÁVEZ, Efrén, GUTIÉRREZ-TORRES, Luis Germán y MARTÍNEZ-LÓPEZ, Fernando José. Desarrollo de una herramienta de software sistema administración de órdenes e inventario (SAO-DiTOTINOS) para restauran DiTOTINOS. Revista de Arquitectura y Diseño. 2019. 3-8: 23-28.

\footnotetext{
*Correspondencia al Autor (Correo Electrónico: e.vega@itsur.edu.mx)

$\dagger$ Investigador contribuyendo como primer autor.
} 


\section{Introducción}

Los sistemas de información en la actualidad son de suma importancia en cualquier contexto, para Andreu, Ricart y Valor (1991), un sistema de información queda definido como: "conjunto formal de procesos que, operando sobre una colección de datos estructurada de acuerdo a las necesidades de la empresa, recopila, elabora y distribuyen selectivamente la información necesaria para la operación de dicha empresa y para las actividades de dirección y control correspondientes, apoyando, al menos en parte, los procesos de toma de decisiones necesarios para desempeñar funciones de negocio de la empresa de acuerdo con su estrategia".

Como se sabe todo sistema de información utiliza como materia prima los datos los cuales son almacenados y procesados para tener una retroalimentación posterior (Hernández Trasobares, 2003), es por ello que el presente proyecto de investigación muestra cómo se controlan las entradas y salidas de productos en almacén para el restaurante DiTotinos con el uso de tecnologías que se reconocen como herramientas de soporte y control según (Akkermans, 2003).

La investigación se planteó con la finalidad de dar una solución al restaurante DiTotinos que se encuentra en el municipio de Moroleón del estado de Guanajuato. La problemática principal a tratar es la forma en la cual se realizaba la actividad de reporteo de productos disponibles en el almacén, actualmente de manera manual, es por ello la sugerencia de un sistema de información para la automatización del proceso actual. Para ello se desarrolló el software SAO-DiTotinos capaz de registrar las entradas y salidas de productos en almacén, teniendo un reporteo confiable. Según (Ochoa Osorio, Pizarro Vázquez, \& Talavera Medina, 2015), en la medida que las empresas controlen y registren conscientemente sus operaciones se conocerá la generación de los costos que le ayudarán a obtener el mayor beneficio posible, minimizar sus costos y apoyando a la toma de decisiones, así como la optimización de tiempos como menciona (Salcedo Guzmán, 2018).
En la actualidad los avances tecnológicos y la innovación tecnológica (en este ámbito las Tecnologías de la Información y la comunicación (TIC's)) han apoyado a la creación de programas de cómputo especializado, los cuales permiten manejar una gran cantidad datos, realizar actividades repetitivas, optimizar tiempos, reducir errores humanos y costos, optimización y mejora de los procesos de servicios. Estas son algunos beneficios que ofrece el software a la medida.

El presente contiene el resultado del proyecto realizado en restaurante y pizzería DiTOTINOS el cual ofrece a sus comensales en el menú además de diversos platillos, las pizzas y bebidas, las órdenes pueden ser en el establecimiento o vía telefónica (servicio a domicilio), se cuenta con un sistema de cómputo para llevar acabo la gestión de pedidos y ventas, dicho software no gestiona el costo y cantidad exacta de la pizza, así como administración del inventario del almacén, teniendo como consecuencia entradas y salidas inciertas. Es por ello que el restaurante DiTOTINOS requiere de un software el cual le permita administrar su inventario de productos y órdenes (ventas), en este contexto se desarrolla el presente trabajo, el cual se realizará en 2 fases (módulo de inventario y módulo 2 órdenes (ventas)) cada fase consta de tres etapas: etapa 1: desarrollo del software, etapa 2: implementación del software y etapa 3: análisis de resultados. Lo anterior tiene como objetivo valorar el impacto de la herramienta de software y contribuir a la optimización de sus recursos y servicios en tiempo óptimo.

\section{Metodología a desarrollar}

El trabajo se desarrolló en el ITSUR con el apoyo de alumnos de la carrera en Ingeniería en Sistemas Computacionales. En el periodo agosto - diciembre 2018 y enero - junio 2019.

Aplicando la siguiente metodología:

Fase 1: desarrollo de módulo de inventario, realizada en agosto - diciembre 2018

En esta fase se aplicaron las siguientes etapas: 
Etapa I: desarrollo del módulo Inventario, para ello se aplicó el ciclo de vida del software el cual consta de:

1.- Determinación de los requerimientos del sistema: aquí se recopilarán los datos que permitirán comprender el ámbito de la información, la funcionalidad, el rendimiento y las interfaces de usuario, se debe de responder las siguientes preguntas claves: ¿Qué es lo que se hace?, ¿Cómo se hace?, ¿Qué tan grande es el volumen de transacciones o decisiones?, ¿Cuál es el grado de eficiencia con el que se efectúa las tareas?, ¿Existe algún problema?, ¿Qué lo origina?, ¿Cuál es la causa?

2.- Diseño del sistema: esta etapa se centra en estructura de los datos, la arquitectura del software, los detalles procedimentales y caracterización de la interfaz de usuario. Para lo cual se realizarán diagramas de casos de uso y diagrama relacional. Para la realización de los diagramas se usará el software StarUML.

3.- Codificación del sistema: en esta etapa se deben de traducir las especificaciones del diseño de forma legible para la computadora, a esto se le conoce como codificación, para este punto se usará lenguaje de programación Visual Studio.NET (C\#).

4.- Pruebas del software: una vez que se genera el código del programa, se inicia la etapa de pruebas, donde se comprobara que al introducir los datos se tiene el resultado esperado. Para ello se aplicará software que permiten procesar y dar seguimiento a las pruebas. Se aplicarán pruebas a los casos de uso.

5.- La implantación: es el proceso de instalar las herramientas de software en nuevos equipos. Para realizar esta tarea es necesario ubicar el servidor e instalar las herramientas de software, se capacitará a los usuarios del software y capturarán los datos pertinentes para el funcionamiento de la aplicación y verificar que en todas las computadoras funcione el software. software.

Etapa II: Implementar la herramienta de
El software se hospedera en un servidor de la empresa, se procesará información de las órdenes. Los resultados guardarán en la base de datos para su análisis posterior, los cuales estarán a disposición del dueño del restaurante.

Etapa III: Determinar el impacto de las herramientas de software.

En esta fase se conocerán los resultados después de aplicar la herramienta de Software y se determinará el impacto al aplicar dicho instrumento, dándonos así a conocer el impacto del sistema de software.

Fase 2: desarrollo del módulo de órdenes (ventas). Se aplicó durante el periodo enero - junio 2019.

$\mathrm{Al}$ igual que la fase anterior se aplicará las tres etapas (desarrollo del módulo de órdenes, implementación del software y determinar el impacto).

\section{Resultados}

Previo a la implementación de las dos etapas se realizó un análisis de datos de tiempo del proceso de reporteo de productos del restaurante DiTotinos se realizó de forma presencial por parte del equipo de trabajo, el cual se dirigió a las instalaciones del restaurante para obtener los datos del tiempo mientras el encargado del almacén realiza su labor en el restaurante DiTotinos durante 3 ocasiones consecutivas, en ese periodo se obtuvieron 3 resultados diferentes. Para medir el tiempo de reporteo fue necesario el uso de un cronometró, que fue usado por una persona del equipo, la actividad se llevó acabo mientras una persona registraba el tiempo en una libreta cuando el usuario realizaba la actividad de reporteo (que se basa en registrar los productos faltantes que no se encuentran en almacén para posteriormente resurtirlos), al terminar de realizar su actividad se detiene el cronometro para obtener el tiempo total. En la tabla 1 se visualizan los tres tiempos obtenidos de la actividad de reporteo.

\begin{tabular}{|r|r|}
\multicolumn{1}{|c|}{ Día } & \multicolumn{2}{c|}{ Tiempo promedio de reporteo } \\
\hline 1 & $00: 27: 31$ \\
\hline 2 & $00: 23: 43$ \\
\hline 3 & $00: 19: 51$ \\
\hline
\end{tabular}

Tabla 1 Tiempos obtenidos 
A continuación, se presentan los resultados de las dos etapas.

En la primera fase desarrollo del módulo Inventario en la etapa de análisis de requerimiento se identificaron los siguientes casos de uso:

- Almacén.

- Inicio de sesión.

- Administración de usuarios.

- Administración de productos.

- Administración de proveedores.

Los cuales son implementados para el desarrollo del software, el cual fue implantado para su uso. La figura 1, representa la interfaz de acceso y la figura 2 la interfaz principal.

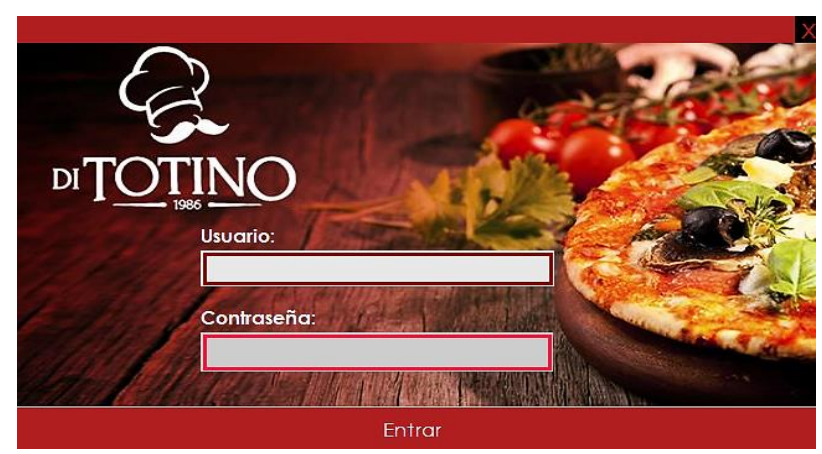

Figura 1 Ventana de acceso módulo de inventario

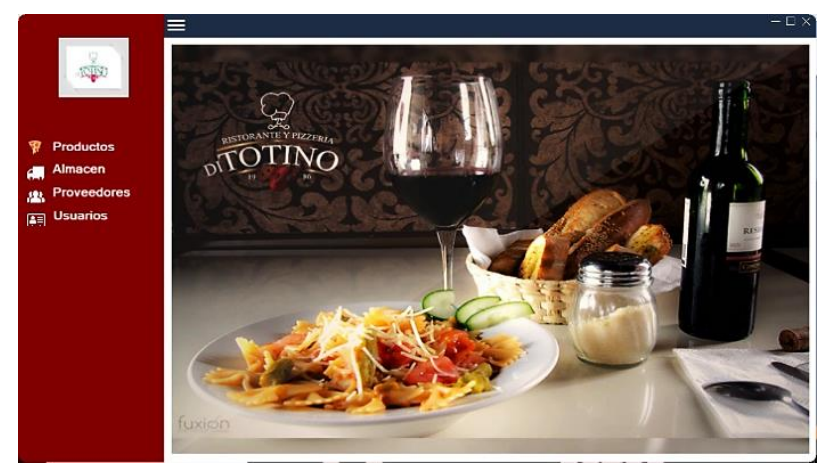

Figura 2 Ventana principal

Para la fase 2, desarrollo del módulo de órdenes (ventas). En la etapa de análisis de requerimientos se establecieron los siguientes casos de uso:

- Inicio de sesión.

- Administrador de usuario.
- Administración de Menú.

- Registro de venta.

- Ventas.

La figura 3 representa un ejemplo de caso de uno ventas registradas, como se puede observar describe el proceso de una orden o venta.

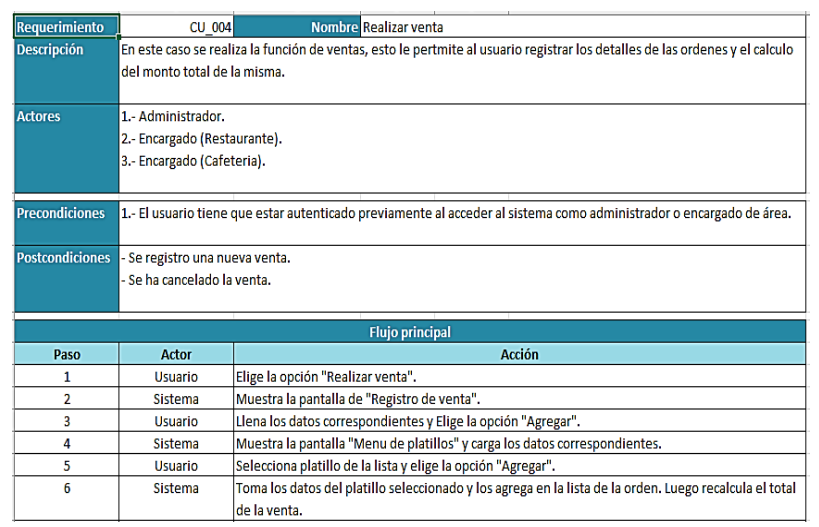

Figura 3 CU_004 Realizar venta

De la fase de implementación se codificaron los casos de uso mencionado anteriormente. La figura 1 presenta el acceso al sistema. Para ello el usuario debe introducir sus datos. La figura 4 presenta la ventana principal del módulo de órdenes(ventas), del lado izquierdo tiene las opciones administrar usuario, administrar menú, la poción de la venta(orden) y ventas registradas.

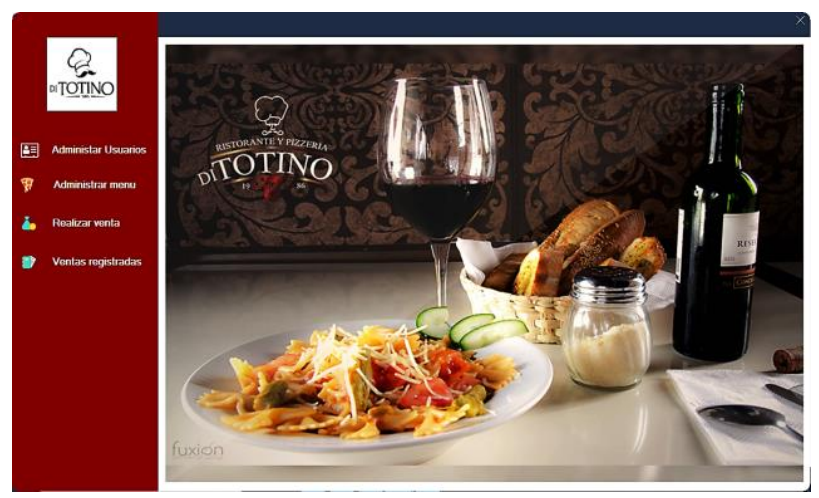

Figura 4 Venta principal de órdenes (ventas)

En la figura 5, se visualiza la ventana de registro de la orden (venta). 


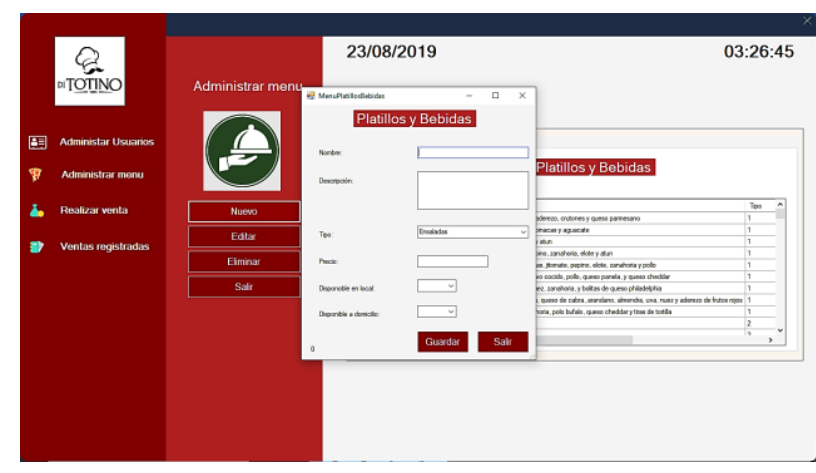

Figura 5 Registro de la ordenes (venta)

Entregado el Sistema SAODiTOTINOS se aplicó una encuesta para determinar la satisfacción del usuario usando formato de encuesta basada en la escala Likert. Los principales aspectos que se consideraron para formular los reactivos fueron los siguientes:

- Eficiencia: Que para fines de la investigación se refiere a medir la rapidez, velocidad, agilidad en que se hace la actividad.

- Eficacia: Se enfoca en la integridad de datos, logro o metas y cantidades precisas.

- Satisfacción: Determina el nivel de conformidad y complacencia del usuario.

La tabla 2, contiene los reactivos de la encuesta, escala Likert.

\begin{tabular}{|c|c|c|c|}
\hline \# & $\begin{array}{c}\text { Facto } \\
\text { r a } \\
\text { evalu } \\
\text { ar }\end{array}$ & Reactivos de la encuesta & \\
\hline 1 & $\begin{array}{l}\text { Efcici } \\
\text { encia }\end{array}$ & $\begin{array}{l}\text { El uso del Software SOA-DiTotinos me hace mas } \\
\text { productivo. }\end{array}$ & $\begin{array}{l}\text { escala } \\
\text { Likert }\end{array}$ \\
\hline 2 & $\begin{array}{l}\text { Efcici } \\
\text { encia }\end{array}$ & $\begin{array}{l}\text { La manera en que se hace el reporteo con el } \\
\text { software SOA-DiTotinos es ágil. }\end{array}$ & $\begin{array}{l}\text { escala } \\
\text { Likert }\end{array}$ \\
\hline 3 & $\begin{array}{l}\text { Efcici } \\
\text { encia }\end{array}$ & $\begin{array}{l}\text { El software disminuyó el tiempo del proceso de } \\
\text { reporteo (conteo al final del dia). }\end{array}$ & $\begin{array}{l}\text { escala } \\
\text { Likert }\end{array}$ \\
\hline 4 & $\begin{array}{l}\text { Eficac } \\
\text { ia }\end{array}$ & $\begin{array}{l}\text { El reporteo (conteo al final del día) con el Software } \\
\text { SOA-DiTotinos es mas preciso que antes. }\end{array}$ & $\begin{array}{l}\text { escala } \\
\text { Likert }\end{array}$ \\
\hline 5 & $\begin{array}{l}\text { Eficac } \\
\text { ia }\end{array}$ & $\begin{array}{l}\text { El Sistema SOA-DiTotinos ayudo a planificar de } \\
\text { mejor manera las compras }\end{array}$ & $\begin{array}{l}\text { escala } \\
\text { Likert }\end{array}$ \\
\hline 6 & $\begin{array}{l}\text { Eficac } \\
\text { ia }\end{array}$ & $\begin{array}{l}\text { El reporte al final del día refleja datos acertados de } \\
\text { los productos que se necesitan }\end{array}$ & $\begin{array}{l}\text { escala } \\
\text { Likert }\end{array}$ \\
\hline 7 & $\begin{array}{l}\text { Satisf } \\
\text { acción }\end{array}$ & $\begin{array}{l}\text { El Sistema SOA-DiTotinos cambió positivamente } \\
\text { la forma en que hago mi trabajo }\end{array}$ & $\begin{array}{l}\text { escala } \\
\text { Likert }\end{array}$ \\
\hline 8 & $\begin{array}{l}\text { Satisf } \\
\text { acción }\end{array}$ & $\begin{array}{l}\text { Es facil de majenar y consultar el Software SOA- } \\
\text { DiTotinos }\end{array}$ & $\begin{array}{l}\text { escala } \\
\text { Likert }\end{array}$ \\
\hline 9 & $\begin{array}{l}\text { Satisf } \\
\text { acción }\end{array}$ & Me siento comodo con el Software SOA-DiTotinos & $\begin{array}{l}\text { escala } \\
\text { Likert }\end{array}$ \\
\hline
\end{tabular}

Tabla 2 Encuesta de satisfacción escala Likert

Analizando el resultado de la encuesta se obtiene los siguiente.

\begin{tabular}{|l|r|l|}
\multicolumn{1}{|c}{ Factor } & \multicolumn{1}{c|}{$\begin{array}{c}\text { Valor } \\
\text { promedio }\end{array}$} & Interpretación \\
\hline Eficiencia & 3.5 & Satisfactoria \\
\hline Eficacia & 4 & Satisfactoria \\
\hline Satisfacción & 3.5 & Satisfactoria \\
\hline
\end{tabular}

Tabla 3 Resultado de la encuesta de satisfacción escala Likert

Con los datos obtenidos de los tiempos en la generación de reporteo se calculó el promedio de datos para poder estimar el tiempo total de los 3 resultados. Una vez obtenido el tiempo total se calculó el coeficiente de variación, también denominado como coeficiente de variación de Spearman. Se aplicó la siguiente fórmula de coeficiente de variación:

$$
C V=\frac{S x}{|x|}
$$

$S_{\mathrm{x}}=$ Desviación típica del conjunto de datos.

$|\mathrm{x}|=$ Valor absoluto de la media del conjunto de datos $\left(X_{1}, X_{2}, \ldots, X_{n}\right)$ y x $\neq 0$.

Como se mencionó el uso del coeficiente de variación, para poder calcularlo, fueron necesarios los datos que se muestran en la tabla 1 , se realizó la suma de los tres tiempos fue de 71.08 minutos, se calculó la media y la desviación estándar, obteniendo los datos mostrados en la Tabla 3.

\begin{tabular}{|c|c|}
\hline Suma total & 71.08 \\
\hline Media: & 23.69 \\
\hline Desviación estándar & 3.2 \\
\hline
\end{tabular}

Tabla 3 Cálculo de la media y desviación estándar

El coeficiente de variación dió como resultado 13.49 que muestra una "variabilidad aceptable" $(10 \%<=\mathrm{CV}<=33 \%)$. Por tanto, se concluyó que los datos son válidos para los cálculos estadísticos.

\section{Agradecimiento}

De antemano agradezco al Sr. Humberto dueño del restaurante DiTotinos, por el apoyo brindado al presente trabajo, a mis compañeros del Cuerpo Académico Fernando José y Luis German. 


\section{Conclusiones}

Con lo mencionado anteriormente se concluye que el objetivo desarrollar un sistema de software (SAO-D'TOTINOS), para valorar el impacto en el restaurante DiTOTINOS. A demás éste estudio permitió comprobar que el uso de dicho software, para el encargado de conteo de productos en almacén, obtuvo una mejora en cuanto al tiempo que toma al momento de checar los productos faltantes el almacén. También, se logró la implementación de la nueva actividad de registro de entradas y salidas de productos del almacén por parte del encargado, que anteriormente no se realizaba.

\section{Referencias}

Andreu, R., Ricart J. E. Y Valor, J. (1991): Estrategia y Sistemas de Información. Mc Graw-Hill, Madrid.

Akkermans, H. B. (2003). "The impact of ERP on supply chain management: Exploratory findings from a European Delphi study. European Journal of Operational Research"

Hernández Trasobares, A. (2003). Los sistemas de información: evolucion y desarrollo. Dialnet, 149-165.

Ochoa Osorio, A. L., Pizarro Vázquez, P. J., \& Talavera Medina, W. M. (2015). "La medicion de los inventarios mejorara la presentacion. Peru".

Salcedo Guzmán, K. E. (2015). Análisis y diseño del sistema para el control de pedidos y pagos del restaurante Las ideas del Chef del cantón Guayaquil año 2014 (Bachelor's thesis, Universidad de Guayaquil. Facultad de Ingeniería Industrial. Carrera de Licenciatura en Sistemas de Información). 